\title{
Short communication: Muscle protein synthetic response to microparticulated whey protein in middle-aged men
}

\author{
C. J. Mitchell, ${ }^{* 1}$ R. F. D’Souza, ${ }^{*}$ A. C. Fanning, † S. D. Poppitt, $\ddagger$ and D. Cameron-Smith* \\ *Liggins Institute, The University of Auckland, Auckland 1142, New Zealand \\ †Fonterra Research and Development Centre, Palmerston North 4442, New Zealand \\ $\ddagger$ School of Biological Sciences, The University of Auckland, Auckland 1142, New Zealand
}

\begin{abstract}
Whey protein concentrate (WPC) is a high-quality dairy ingredient that is often included in formulated food products designed to stimulate muscle anabolism. Whey protein concentrate can be affected by UHT processing, and its sensory properties are not compatible with some formulated food products. Microparticulated WPC (mWPC) is a novel ingredient that is resistant to heat treatment and has enhanced sensory properties. When 16 healthy middle-aged men consumed $20 \mathrm{~g}$ of either WPC or mWPC, both proteins increased plasma essential AA and leucine concentrations with no detectable difference in curve kinetics. Myofibrillar protein synthesis was increased in both groups for 90 min after ingestion with no difference between groups. Ingestion of $\mathrm{mWPC}$ resulted in a muscle anabolic response that was equivalent to that of WPC over the full 210-min measurement period. Formulated products incorporating $\mathrm{mWPC}$ or standard WPC would provoke equivalent anabolic responses.
\end{abstract}

Key words: muscle protein turnover, dairy protein, protein ingredient, protein concentrate

\section{Short Communication}

Muscle mass is an important determinant of metabolic health and physical function (von Haehling et al., 2010). A gradual loss of muscle mass and function, termed sarcopenia, begins in middle age and is partially underpinned by a diminished muscle protein synthetic response to protein feeding (anabolic resistance; Churchward-Venne et al., 2014). The consumption of additional high-quality protein is known to stimulate muscle protein synthesis (MPS) and to help overcome anabolic resistance (Moore et al., 2015). Whey protein is a high-value dairy protein fraction that is rich in EAA, especially leucine. It is soluble at low $\mathrm{pH}$, and

Received November 9, 2016.

Accepted March 2, 2017.

${ }^{1}$ Corresponding author: cameron.mitchell@auckland.ac.nz its ingestion results in rapid aminoacidemia (Farnfield et al., 2009). Many studies have compared the effects of whey protein ingestion at rest or after exercise with ingestion of other animal- and plant-based protein sources and found that whey induced a superior MPS response (van Vliet et al., 2015). Although some studies have shown that in certain situations other protein sources, including milk protein concentrate, may induce an equivalent MPS response (Mitchell et al., 2015a), to date no studies have demonstrated a superior protein source.

Whey protein is a term used generically to describe the soluble proteins isolated from milk. Cheese or acid whey substrates are typically used to create whey-based ingredients (Lagrange et al., 2015). Whey protein concentrate (WPC) is a common food ingredient used to increase the protein content of formulated products and is often added to products designed to support muscle health and simulate MPS (Lagrange et al., 2015). Whey protein concentrate is not an ideal additive for all formulated products because of its sensitivity to UHT processing, which may result in protein denaturation, aggregation, and flocculation. This can be problematic in the formulation of ready-to-drink beverages. Microparticulation is an advanced processing technology that is typically achieved by thermal aggregation and acid precipitation combined with high shear conditions (Renard et al., 2002). This can improve heat stability and prevent aggregation and gelation in consumer products in which UHT processing is required (ÇakırFuller, 2015). Microparticulated WPC (mWPC) also has increased stability at a lower $\mathrm{pH}$ (Dissanayake et al., 2012) and a unique mouth feel, which makes it suitable to replace fat in many formulated products (Civille, 1990).

The development of mWPC allows for the manufacture of a wider variety of consumer products. However, it is unknown whether the microparticulation of whey protein affects plasma AA kinetics and the postprandial MPS response. The purpose of the present study was to investigate the plasma AA and MPS response to the ingestion of $20 \mathrm{~g}$ of standard WPC or mWPC 
that has not been subjected to secondary processing in healthy middle-aged men.

Sixteen healthy sedentary to recreationally active males provided informed consent to participate in this trial and were randomized to either WPC $(52.6 \pm 3.9$ yr, $\left.26.7 \pm 2.5 \mathrm{~kg} / \mathrm{m}^{2}, \mathrm{n}=8\right)$ or $\operatorname{mWPC}(51.0 \pm 3.5$ yr, $26.5 \pm 3.1 \mathrm{~kg} / \mathrm{m}^{2}, \mathrm{n}=8$ ) in a parallel design. The study was approved by the Northern Health and Disability Ethics Committee (New Zealand). The results from the WPC group have been previously reported by our research team (Mitchell et al., 2015a). Middle-aged men were chosen because this life stage likely represents the start of a transition from anabolic sensitivity to anabolic resistance and may be an ideal time period to target interventions to preserve muscle mass (Mitchell et al., 2016).

Fasted participants underwent a primed constant infusion of ${ }^{13} \mathrm{C}_{6}$ phenylalanine with serial muscle biopsies and blood draws. The fractional synthetic rate (FSR) of myofibrillar muscle proteins was calculated at rest and for 90 and 210 min after beverage ingestion as previously described (Mitchell et al., 2015a). This time period was chosen to capture the postprandial period and to provide maximal temporal resolution given the sensitivity of the methods used. Plasma AA concentrations were measured for $210 \mathrm{~min}$ after beverage ingestion as described by Milan et al. (2015).

Participants were randomly assigned to consume either $20 \mathrm{~g}$ of WPC (WPC 392, Fonterra Co-Operative Group Ltd., Auckland, New Zealand) or $20 \mathrm{~g}$ of mWPC (WPC 550, Fonterra Co-Operative Group Ltd.) in a double-blind fashion. Each ingredient was dissolved in $350 \mathrm{~mL}$ of water and contained less than $2 \mathrm{~g}$ of fat and approximately $2 \mathrm{~g}$ of carbohydrate (Table 1). Neither beverage was subjected to UHT processing or any other secondary processing.

Table 1. Beverage macronutrient and EAA composition of whey protein concentrate (WPC) and microparticulated WPC (mWPC)

\begin{tabular}{lcc}
\hline Item & WPC & mWPC \\
\hline Energy $(\mathrm{kJ})_{\text {Protein }}^{1}(\mathrm{~g})$ & 421 & 412 \\
Carbohydrate, lactose $(\mathrm{g})_{\text {Fat }(\mathrm{g})}$ & 20 & 20 \\
EAA $(\mathrm{g})$ & 2.1 & 1.8 \\
Threonine & 1.2 & 1.2 \\
Valine & & \\
Isoleucine & 1.0 & 1.6 \\
Leucine & 1.3 & 1.2 \\
Phenylalanine & 1.1 & 1.4 \\
Lysine & 2.1 & 2.3 \\
Histidine & 1.0 & 0.7 \\
Methionine & 1.9 & 1.9 \\
Tryptophan & 0.6 & 0.4 \\
\hline
\end{tabular}

${ }^{1}$ Protein calculated as $6.25 \times$ total nitrogen.
Two-way ANOVA with time as a within-subject factor and group as a between-subjects factor was used for all analyses, and Sidak's post hoc procedure was used where appropriate. All analyses were conducted using Graphpad Prism (version 6.0; La Jolla, CA). Data are shown as mean \pm standard deviation in the text and \pm standard error of the mean in the figures. Alpha was set at $P<0.05$. The threshold for bioequivalence was set at $<20 \%$ difference between beverages as described in Walker and Nowacki (2011).

Concentrations of plasma leucine, branched chain AA, EAA, and total AA significantly increased after beverage consumption to a maximum around $1 \mathrm{~h}$ postprandially (time, $P<0.05$ ), but with no differences between groups (Figure 1). Myofibrillar FSR was increased by protein feeding regardless of source for 90 $\min (P=0.013)$ after feeding and returned to baseline in the 90 - to 210 -min period $(P=0.570)$ with no interaction between groups $(P=0.383$; Figure $2 \mathrm{~A})$. The aggregate FSR for the full 210-min postfeeding period was also elevated with no significant differences between groups $(P=0.993$; Figure $2 \mathrm{~B})$. The upper and lower equivalence thresholds were 0.04474 and $0.02983 \% / \mathrm{h}$, respectively, based on a $20 \%$ variation from the mean aggregate MPS response achieved in the WPC group. The $95 \%$ confidence interval of the aggregate response was 0.02987 to $0.04336 \% / \mathrm{h}$, thus establishing equivalence of aggregate MPS response $(P<0.025)$.

The current study showed for the first time that the ingestion of $20 \mathrm{~g}$ of $\mathrm{mWPC}$ resulted in a MPS response equivalent to the ingestion of $20 \mathrm{~g}$ of standard WPC over a 210-min incorporation period. This finding is not surprising given the almost identical kinetics of the EAA, particularly leucine, in plasma. Previous studies that have shown divergent MPS responses to the same dose of protein suggest that the leucine content of the protein and the speed at which the AA appear in the blood are the primary factors that determine the magnitude of the MPS response (Katsanos et al., 2006; West et al., 2011; van Vliet et al., 2015). Both $\mathrm{mWPC}$ and WPC have very similar leucine contents and plasma AA appearance, which likely is primarily responsible for our findings. In the rested state it appears that the dose of leucine in a given protein is more important than the rate of aminoacidemia in determining the MPS response (Mitchell et al., 2015a,b). The similar and rapid aminoacidemia induced by both $\mathrm{mWPC}$ and WPC may be more relevant in situations in which the muscle has previously been sensitized to feeding, such as after exercise (West et al., 2011; Burd et al., 2012). Although statistical significance was not approached, FSR was slightly higher from 0 to 90 min in the WPC group and from 90 to $210 \mathrm{~min}$ in the mWPC group, suggesting that microparticulation 


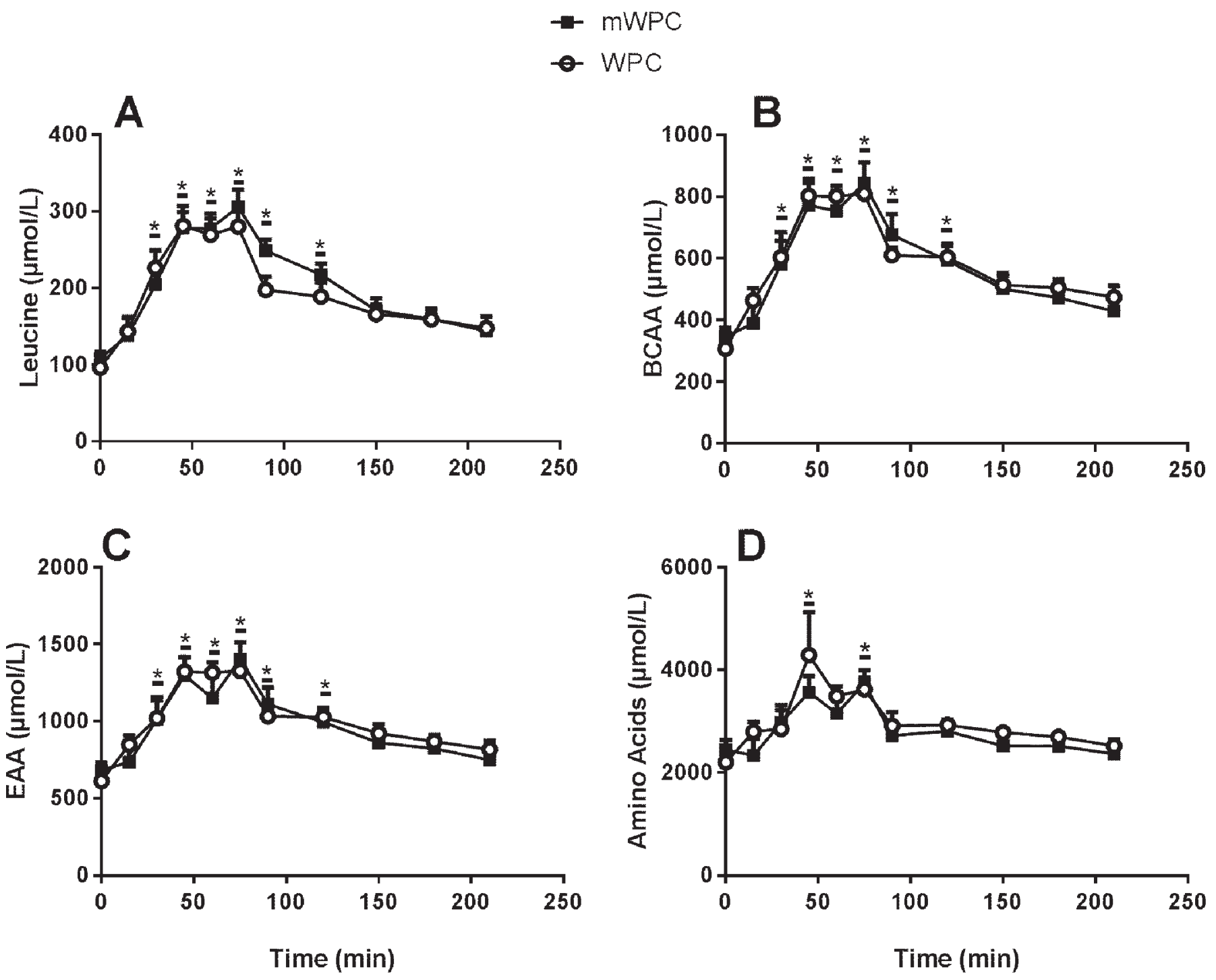

Figure 1. Plasma concentrations of (A) leucine, (B) branched-chain AA (BCAA), (C) EAA, and (D) total AA. WPC = whey protein concentrate; $\mathrm{mWPC}=$ microparticulated WPC. Horizontal lines represent main effects for time. ${ }^{*}$ Significantly different from time $0(P<0.05)$. Error bars represent mean \pm SEM. Eight subjects per group were measured at each time point.

could have a minor effect on the time course of the MPS response. However, the practical significance of this finding is likely minor.

The protein dose used in this study was chosen because it represents the minimal dose required to elicit a maximal MPS in young men (Witard et al., 2014) and older men (Yang et al., 2012) at rest. However, some studies have suggested that an even larger protein dose may be required to maximally stimulate MPS in elderly men (Moore et al., 2015). It is possible that a divergent MPS response may have been observed had a smaller suboptimal dose been used. However, given the similar leucine content and protein kinetics we observed, this is unlikely.
The current research compared the effect of WPC or mWPC consumption on MPS using a relatively simple beverage model: whey protein powder rehydrated in water. This comparison does not allow for delineation of any effect of secondary processing conditions, such as UHT processing. However, it was selected because the WPC product would gel and aggregate following UHT processing, creating an impractical beverage. The lack of a group that consumed mWPC following UHT processing is a limitation of the study. Previous work has shown that microparticulation prevents aggregation and gelation of WPC induced by heat treatment (Çakır-Fuller, 2015), but it is not known how the AA kinetics or MPS response to $\mathrm{mWPC}$ is affected by heat 
treatment. Ultra-high temperature processing of milk results in a slightly lower nitrogen retention compared with either microfiltered or pasteurized milk; however, this finding was apparent over an 8-h postprandial period and may have limited relevance to individuals following a normal dietary pattern (Lacroix et al., 2008). Future research could address the effects of secondary UHT processing of $\mathrm{mWPC}$ on AA kinetics and MPS.

Ingestion of $20 \mathrm{~g}$ of microparticulated whey protein resulted in an MPS response equivalent to the ingestion of standard whey protein in healthy middle-aged men, and this finding was mediated by similar AA kinetics between the 2 proteins. Formulated products containing mWPC would have a similar effect on muscle anabo-
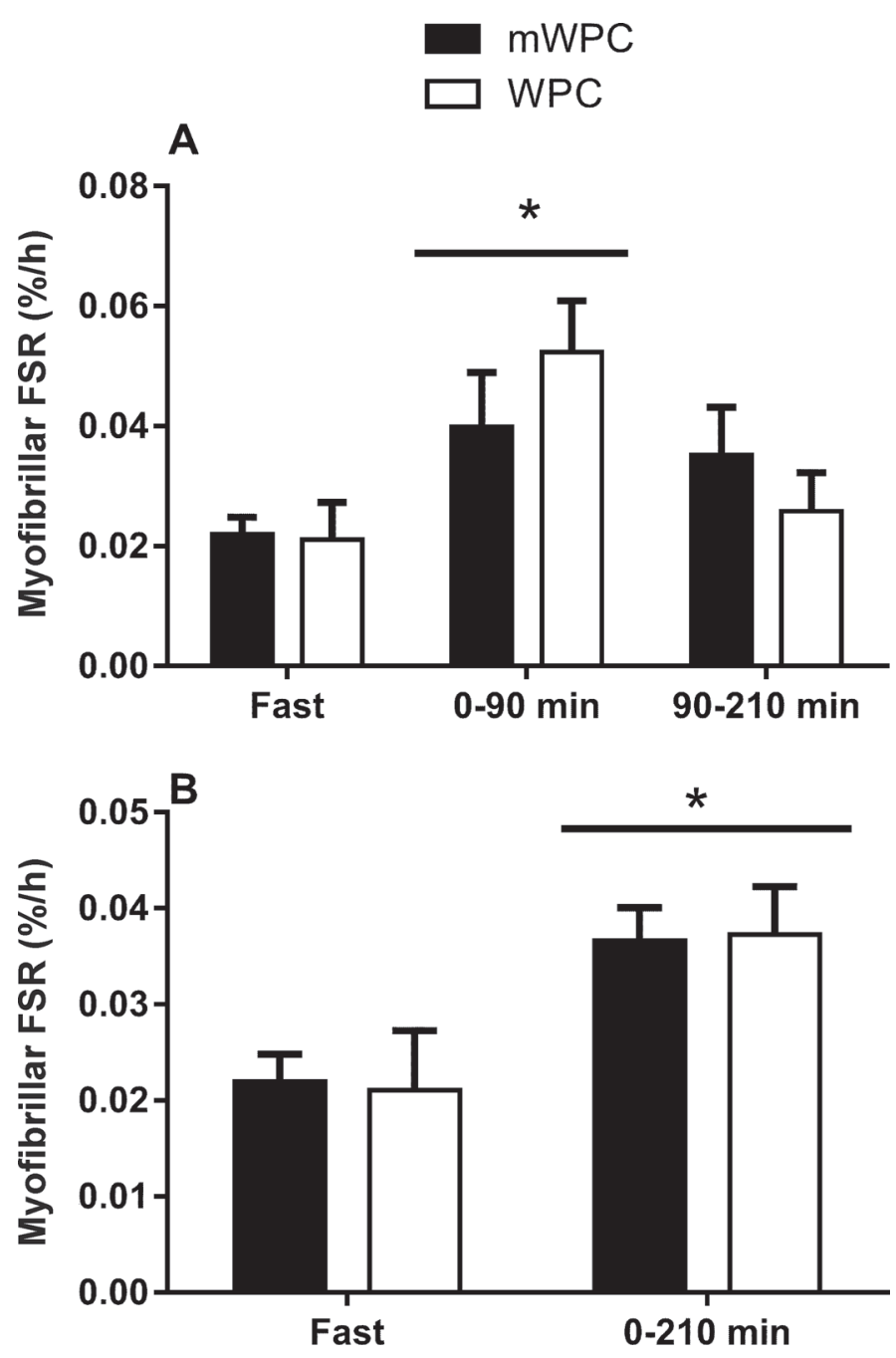

Figure 2. Myofibrillar protein synthesis: (A) time course and (B) aggregate myofibrillar fractional synthetic rate (FSR) in response to intake of $20 \mathrm{~g}$ of protein. $\mathrm{WPC}=$ whey protein concentrate: $\mathrm{mWPC}$ $=$ microparticulated WPC; Fast $=$ fasted sample. Horizontal lines represent main effects for time. ${ }^{*}$ Significantly different from time $0(P<$ 0.05). Error bars represent mean \pm SEM. Eight subjects per group were measured at each time point. lism as the same dose of WPC. However, the effect of secondary processing of consumer products would need to be established separately.

\section{ACKNOWLEDGMENTS}

We thank the Liggins Institute Clinical Research Unit staff as well as all the participants for their time commitment. CJM and DCS received financial support from the New Zealand Primary Growth Partnership post-farm gate program, funded by Fonterra Co-operative Group Ltd. (Auckland, New Zealand) and the New Zealand Ministry for Primary Industries (Wellington New Zealand), to conduct this study. SDP is the Fonterra chair in human nutrition, University of Auckland. $\mathrm{ACF}$ is a current employee of Fonterra Co-operative Group Ltd.

\section{REFERENCES}

Burd, N. A., Y. Yang, D. R. Moore, J. E. Tang, M. A. Tarnopolsky, and S. M. Phillips. 2012. Greater stimulation of myofibrillar protein synthesis with ingestion of whey protein isolate v. micellar casein at rest and after resistance exercise in elderly men. Br. J. Nutr. 108:958-962.

Cakır-Fuller, E. 2015. Enhanced heat stability of high protein emulsion systems provided by microparticulated whey proteins. Food Hydrocoll. 47:41-50.

Churchward-Venne, T. A., L. Breen, and S. M. Phillips. 2014. Alterations in human muscle protein metabolism with aging: Protein and exercise as countermeasures to offset sarcopenia. BioFactors 40:199-205.

Civille, G. V. 1990. The sensory properties of products made with microparticulated protein. J. Am. Coll. Nutr. 9:427-430.

Dissanayake, M., S. Liyanaarachchi, and T. Vasiljevic. 2012. Functional properties of whey proteins microparticulated at low $\mathrm{pH}$. J. Dairy Sci. 95:1667-1679.

Farnfield, M. M., C. Trenerry, K. A. Carey, and D. Cameron-Smith. 2009. Plasma amino acid response after ingestion of different whey protein fractions. Int. J. Food Sci. Nutr. 60:476-486.

Katsanos, C. S., H. Kobayashi, M. Sheffield-Moore, A. Aarsland, and R. R. Wolfe. 2006. A high proportion of leucine is required for optimal stimulation of the rate of muscle protein synthesis by essential amino acids in the elderly. Am. J. Physiol. Endocrinol. Metab. 291:E381-E387.

Lacroix, M., C. Bon, C. Bos, J. Leonil, R. Benamouzig, C. Luengo, J. Fauquant, D. Tome, and C. Gaudichon. 2008. Ultra high temperature treatment, but not pasteurization, affects the postprandial kinetics of milk proteins in humans. J. Nutr. 138:2342-2347.

Lagrange, V., D. Whitsett, and C. Burris. 2015. Global market for dairy proteins. J. Food Sci. 80(Suppl. 1):A16-A22.

Milan, A. M., R. F. D'Souza, S. Pundir, C. A. Pileggi, M. P. G. Barnett, J. F. Markworth, D. Cameron-Smith, and C. Mitchell. 2015. Older adults have delayed amino acid absorption after a high protein mixed breakfast meal. J. Nutr. Health Aging 19:839-845.

Mitchell, C. J., R. F. D'Souza, N. Zeng, R. A. McGregor, A. C. Fanning, S. D. Poppitt, and D. Cameron-Smith. 2016. Understanding the sensitivity of muscle protein synthesis to dairy protein in middle-aged men. Int. Dairy J. 63:35-41.

Mitchell, C. J., R. A. McGregor, R. F. D'Souza, E. B. Thorstensen, J. F. Markworth, A. C. Fanning, S. D. Poppitt, and D. CameronSmith. 2015a. Consumption of milk protein or whey protein results in a similar increase in muscle protein synthesis in middle aged men. Nutrients 7:8685-8699. 
Mitchell, W. K., B. E. Phillips, J. P. Williams, D. Rankin, J. N. Lund, K. Smith, and P. J. Atherton. 2015b. A dose- rather than delivery profile-dependent mechanism regulates the "muscle-full" effect in response to oral essential amino acid intake in young men. J. Nutr. $145: 207-214$

Moore, D. R., T. A. Churchward-Venne, O. Witard, L. Breen, N. A Burd, K. D. Tipton, and S. M. Phillips. 2015. Protein ingestion to stimulate myofibrillar protein synthesis requires greater relative protein intakes in healthy older versus younger men. J. Gerontol. A Biol. Sci. Med. Sci. 70:57-62.

Renard, D., L. Lavenant, C. Sanchez, Y. Hemar, and D. Horne 2002. Heat-induced flocculation of microparticulated whey proteins (MWP); Consequences for mixed gels made of MWP and $\beta$-lactoglobulin. Colloids Surf. B Biointerfaces 24:73-85.

van Vliet, S., N. A. Burd, and L. J. van Loon. 2015. The skeletal muscle anabolic response to plant- versus animal-based protein consumption. J. Nutr. 145:1981-1991.

von Haehling, S., J. E. Morley, and S. D. Anker. 2010. An overview of sarcopenia: Facts and numbers on prevalence and clinical impact. J. Cachexia Sarcopenia Muscle 1:129-133.
Walker, E., and A. S. Nowacki. 2011. Understanding equivalence and noninferiority testing. J. Gen. Intern. Med. 26:192-196.

West, D. W., N. A. Burd, V. G. Coffey, S. K. Baker, L. M. Burke, J. A. Hawley, D. R. Moore, T. Stellingwerff, and S. M. Phillips. 2011 Rapid aminoacidemia enhances myofibrillar protein synthesis and anabolic intramuscular signaling responses after resistance exercise. Am. J. Clin. Nutr. 94:795-803.

Witard, O. C., S. R. Jackman, L. Breen, K. Smith, A. Selby, and K. D. Tipton. 2014. Myofibrillar muscle protein synthesis rates subsequent to a meal in response to increasing doses of whey protein at rest and after resistance exercise. Am. J. Clin. Nutr. 99:86-95.

Yang, Y., L. Breen, N. A. Burd, A. J. Hector, T. A. Churchward-Venne, A. R. Josse, M. A. Tarnopolsky, and S. M. Phillips. 2012. Resistance exercise enhances myofibrillar protein synthesis with graded intakes of whey protein in older men. Br. J. Nutr. 108:1780-1788. 